\title{
1)Adaptive spectral band identification method in noncontact temperature measurement based on dynamic programming
}

\author{
Hui Wang, Dejiang Chen, Qingtao Yang, Jun Zhang, Liping Liu \\ (Hypervelocity Aerodynamics Institute, China Aerodynamics Research and Development Center, \\ Mianyang, Sichuan, 621000, China)
}

Stefan Löhle

(High Enthalpy Flow Diagnostics Group, Institute of Space Systems, University of Stuttgart, 70569 Stuttgart, Germanny)

\begin{abstract}
An adaptive method based on dynamic programming is proposed to identify the spectral band for non-continuous measurement of surface temperature of heatshield materials when Fourier transform infrared (FTIR) spectrometer is used to collect the radiation spectrum in the dynamic heating environment of a high-frequency plasma wind tunnel. First, the radiation spectrum is converted to a time series. Then, high-frequency parts of the measurement spectral signal are obtained by multi-resolution analysis of one-dimensional discrete wavelet and then the suitable spectral band required by a non-contact temperature measurement is adaptively identified based on dynamic programming. Eventually, surface temperature and its corresponding emissivity can be determined. Results of the experiment conducted on a benchmark material (graphite) in the dynamic heating environment of high frequency plasma wind tunnel show the proposed method to be practical.
\end{abstract}

Key Words: plasma; radiation spectrum; noncontact temperature measurement; adaptive spectral band identification

\section{Introduction}

Thermal protection materials experience an enormous heating procedure when the vehicle re-enters the Earth's atmosphere. High surface temperatures at high emissivity of non-ablation type of material are advantageous to enhance the thermal dissipation by radiation. So it's necessary to analyze the surface temperature and its emissivity to assess its heat dissipation performance in the high-frequency plasma wind-tunnel.

The thermal protection experiment in the high-frequency plasma wind-tunnel is a dynamical procedure and FTIR is used to dynamically obtain normal spectral irradiance for a series of surface temperatures and emissivities in the dynamical heating conditions. According to the energy distribution of spectral radiation,

* Corresponding author. Tel: +86 8162364034 ;

Fax: +86 8162362490 ;

E-mail address: wang_sunshine@163.com the non-contact temperature measurement method is applied to obtain the corresponding surface temperature, and then the emissivity can be derived under dynamic heating conditions ${ }^{[1]}$.

Recently, most of the non-contact temperature measurement methods are based on two or multi wavelength radiation spectra ${ }^{[2-5]}$. The signals collected by single color or two color pyrometers are obtained as an integral in a narrow-band spectral irradiance. The ripple of the single color signal history or between two-color signals is not considered, which means that temperature measurement procedure is robust, but a mean value. However, due to high-resolution performance of the Fourier transform infrared (FTIR), the spectrum signals are obtained. But, there are no existing integral operations in these narrow-band spectral irradiances. Therefore, spectral signals have obviously small ripples, which will degrade the temperature measurement results based on two or multi-spectral wavelength method ${ }^{[1]}$. Espe- 
cially accounting for different frequency characteristics with different spectral bands about the ripple under plasma background radiation in the dynamic heating environment, to the authors' knowledge, no literature is found on how to identify spectral bands for measurements to improve the fidelity of the non-contact temperature measurement method based on FTIR, though there are reports on testing emissivity by spectrometer in the static heating environments without plasma radiation disturb and the surface temperature is measured by contact methods or pyrometer ${ }^{[6-11]}$. In addition to that, in order to avoid the plasma radiation, the external pyrometer with a defined measuring wavelength is used to obtain the irradiance form the front surface of sample and it's true temperature is measured by a pyrometer based on the sample backside formed similar blackbody cavity assumption during the sample is reached to stable heating state ${ }^{[12]}$.

Account obtaining multi spectral emissivities during dynamical heating procedure of plasma wind tunnel, varying surface temperature of sample should be deduced Synchronously from the spectral signal of FTIR spectrometer. Due to the problem that there is more or less drastic background radiation and much interference between different spectral bands, an adaptive spectral band identification method is proposed to obtain the required spectral band by combining the dynamic programming method and multi-resolution analysis of one-dimension discrete wavelet for non-contact surface temperature measurement in the dynamic heating environment of plasma wind tunnel. The proposed method is applied to process a practical experiment and the results show that the proposed method is effective and practical.

In the next section, due to existing significant plasma disturbance in the original spectral signal from thermal radiation of the front surface of heating sample, the Preprocessing method of the plasma radiation spectrum is firstly described. Then, Adaptive spectral band identification based on dynamic programming is proposed in Sec. III. Finally, experimental setup is briefly presented and experimental processing and identified results are given; besides, the emissivity of reference material, graphite, is measured from these dynamic varying surface temperature obtained based the above discussed adaptive spectral band identification results in Sec. IV.

\section{Preprocessing the plasma radiation spectrum}

The measurement of the thermal radiation characteristics of a heatshield material in a plasma wind tunnel depends on the general radiation spectrum of the test model disturbed by the spectrum of the high-temperature plasma flow. So the original spectrum should be preprocessed to subtract the plasma spectrum as far as possible by the energy superposition principle. Then

$$
\begin{aligned}
S_{p}(\lambda) & =S_{0}(\lambda)-S_{b}(\lambda) \\
S^{\prime}(\lambda) & =S(\lambda)-S_{p}(\lambda)
\end{aligned}
$$

Where $S_{0}$ is the radiation spectrum of the plasma flow without test model; $S_{p}$ is the radiation spectrum of the pure plasma without ambient background radiation. $S_{b}$ is the ambient background radiation spectrum without the plasma flow; $S$ is the radiation spectrum of test model with radiation perturbation. $S^{\prime}$ is the radiation spectrum of test model without plasma radiation perturbation, which is finally obtained based on Eq. (1) and Eq.(2).

\section{Adaptive spectral band identification based on dynamic programming}

The above discussed preprocessing method is effective to remove most part of the spectrum of high-temperature plasma flow, however, some ripple still exists in different bands due to the plasma instability. This ripple is significant for noncontact temperature measurement based on FTIR spectroscopy. Moreover, the plasma dynamics have different influence on the thermal radiation of test model at different time and spectral bands. Vapor and carbon dioxide have different absorbency ability at different spectral bands, which result in different disturbing frequencies and amplitude. So 
the steady, long and near-short wavelength spectral band without obvious ripple should be adaptively chosen to calculate surface temperatures.

Dynamic programming focuses on time-ordered decision-making problems. Therefore, by introducing time sequence, dynamic programming problems can be treated as multi-stage decision-making problems ${ }^{[13,14]}$. If a problem can be divided into some sequence stages and one decision should be made at each stage, so all of the decisions form a series, and the whole procedure is viewed as time-ordered decision-making problem. Considering the mean and mean square, the total cost function ${ }^{[15]}$ is denoted as follows.

$$
\begin{aligned}
(\hat{\boldsymbol{\tau}}, \hat{\boldsymbol{\theta}}) & =\arg \min _{(\tau, \boldsymbol{\theta}) \in \Gamma_{K} \times \Theta_{K}}\left\{\frac{1}{n} \sum_{k=1}^{K} W_{k}\right\} \\
& =\arg \min _{(\tau, \boldsymbol{\theta}) \in \Gamma_{K} \times \Theta_{K}}\left\{\frac{1}{n} \sum_{k=1}^{K}\left(\frac{\left\|\mathbf{Y}_{k}-\mu_{k}\right\|^{2}}{\sigma_{k}^{2}}+n_{k} \log \sigma_{k}^{2}\right)\right\}
\end{aligned}
$$

Here, $W_{k}$ is the cost function at the $k^{\text {th }}$ band, $K$ is the number of bands, $n$ is the number of total data points, $\mu_{k}$ is the mean at the $k^{\text {th }}$ band, $\sigma_{k}^{2}$ is the mean square at the $k^{\text {th }} \quad$ band. $\boldsymbol{\theta}=\left(\mu_{k}, \sigma_{k}^{2}\right)_{k=1, \cdots, K}$, $\boldsymbol{\tau}=\left[\tau_{1}, \tau_{2}, \cdots \tau_{K-1}\right]$ is the $(k-1)^{\mathrm{th}}$ partition point corresponding to the $k^{\text {th }}$ band. The data series $\mathbf{Y}_{k}=\left[Y_{\tau_{k-1}+1}, Y_{\tau_{k-1}+2} \cdots Y_{\tau_{k}}\right]$ at the $k^{\text {th }}$ band and the coordinate is unitary, namely $\tau_{0}=0, \tau_{K}=1 . n_{k}$ is the number of data points at the $k^{\text {th }}$ band, namely $n_{k}=\tau_{k}-\tau_{k-1} \cdot \Gamma_{K} \subset \mathbf{R}^{K-1}, \Theta_{K} \subset R^{K} \times R^{K}$.

In this study, the discrete calibrated spectrum irradiance obtained by FTIR $^{[1]}$ is treated as one set of time series sequence data. Only after the dynamic programming is applied to obtain the desired time series band, the time band should be converted into the corresponding spectral band by simple linear proportional relationship. And the dynamic programming method is illustrated in detail as follows.

1) Establish the data series of dynamic programing

Suppose the set of discrete spectral irradiance da- ta, $\bar{y}=\left[y_{1}, y_{2} \cdots y_{N}\right]$. Then, it is decomposed into 4 components by $\mathrm{db} 3$ wavelet. Considering a suitable band for noncontact temperature measurement, plasma and background radiation becomes obvious at the high-frequency band, so reconstruct the high-frequency $4^{\text {th }}$-level wavelet coefficient and it is denoted as $\bar{y}_{d, 4}=\left[y_{d, 1}, y_{d, 2} \cdots y_{d, N}\right]$. The detailed procedure on wavelet multi-resolution analysis and reconstruction of signal can be learn about in the reference [16]. Then , based on above signal processing procedure, the data series used for dynamic programming can be constructed as:

$$
\mathbf{Y}_{N}=\left[Y_{1}, Y_{2} \cdots, Y_{N}\right]
$$

Where, $Y_{i}=y_{d, i}-\sum_{j=1}^{N} y_{d, j} / N, i=1,2, \cdots, N$. And the minimal interval is $d$ between the near partition points, the maximal number of partition points is $K$.

2) Only one partition point, $C^{1}(1)$ (the first partition scheme)

In this step, the mean equals to 0 based on Eq. (4) and then only the mean square is considered. So Eq. (3) can be simplified and rewritten with the starting points $Y_{i}$ and $m$ groups of data series as following,

$$
W_{i}=D_{i, m}=m \ln \left(\sum_{j=0}^{m-1} Y_{(i+j)}^{2} / m\right)
$$

Thus the sum of sub-bands' cost functions has its minimal value $I_{N-j}^{1}(1)$ and the optimal partition point $C_{N-j}^{1}(1)$ can be determined respectively by

$$
\begin{gathered}
I_{N-j}^{1}(1)=\min _{0 \leq i \leq(N-j-2 d-1)}\left\{D_{1, d+i}+D_{d+i+1, N-j-d-i}\right\}, \\
j=0,1,2, \cdots,(N-2 d) \\
C_{N-j}^{1}(1)=d+i^{*} j=0,1,2, \cdots,(N-2 d)
\end{gathered}
$$

Where,

$$
i^{*}=\arg \min _{0 \leq i \leq(N-j-2 d-1)}\left\{D_{1, d+i}+D_{d+i+1, N-j-d-i}\right\}
$$


3 ) two partition points $\left\{C^{2}(i), 1 \leq i \leq 2\right\}$ (the second partition scheme)

In the band $\mathbf{Y}_{N-j}=\left[Y_{1}, Y_{2} \cdots Y_{N-j}\right]$, the minimal of cost functions' sum of the sub-bands at the right of the second partition point and the optimal partition point $C_{N-j}^{2}(1)$ are expressed as follows, respectively, with the aid of the minimal value of cost functions' sum of the sub-bands at the left of the first partition point in the first scheme.

$$
\begin{aligned}
I_{N-j}^{2}(1)= & \min _{0 \leq i \leq(N-j-3 d-1)}\left\{I_{2 d+i}^{1}(1)+D_{2 d+i+1, N-j-2 d-i}\right\} \\
& , \quad j=0,1,2, \cdots,(N-3 d) \\
C_{N-j}^{2}(1)= & 2 d+i^{*}, \quad j=0,1,2, \cdots,(N-3 d)
\end{aligned}
$$

Where,

$$
i^{*}=\arg \min _{0 \leq i \leq(N-j-3 d-1)}\left\{I_{2 d+i}+D_{2 d+i+1, N-j-2 d-i}\right\}
$$

When the first partition point is determined, the second partition point can be confirmed in the band $\mathbf{Y}_{C_{N-j}^{2}(1)}=\left[Y_{1}, Y_{2} \cdots Y_{C_{N-j}^{2}(1)}\right]$ as follows.

$$
C_{C_{N-j}^{2}(1)}^{2}(2)=C_{C_{N-j}^{2}(1)}^{1}(1)
$$

4) $k$ partition points (the kth partition scheme)

In the band $\mathbf{Y}_{N-j}=\left[Y_{1}, Y_{2} \cdots Y_{N-j}\right]$, the minimal of cost functions' sum of the sub-bands at the right of the first partition point and the optimal partition point $C_{N-j}^{k}(1)$ are expressed as follows, respectively, with the aid of the minimal value of cost functions' sum of the sub-bands at the left of the first partition point in the $(k-1)$ scheme, whose procedure is similar with the first and second schemes.

$$
\begin{gathered}
I_{N-j}^{k}(1)=\min _{0 \leq i \leq(N-j-(k+1) d-1)}\left\{I_{k d+i}^{k-1}(1)+D_{k d+i+1, N-j-k d-i}\right\}, \\
j=0,1,2, \cdots,(N-(k+1) d) \\
C_{N-j}^{k}(1)=k d+i^{*}, \quad j=0,1,2, \cdots,(N-(k+1) d)
\end{gathered}
$$

Here, $i^{*}=\arg \min _{0 \leq i \leq(N-j-(k+1) d-1)}\left\{I_{k d+i}^{k-1}(1)+D_{k d+i+1, N-j-k d-i}\right\}$

Similarly, other optimal partition points $M_{k}(j)$ can be determined with the aid of the minimal values determined in the former $k-1$ cases by the one-by-one iteration algorithm.

$M_{k}(j)=C_{M_{k}(j-1)}^{k}(j)=C_{M_{k}(j-1)}^{k-j+1}(1), j=2,3, \cdots, k$ (13) Where $M_{k}(1)=C_{N}^{k}(1)$.

5 ) the optimal partition points number determination

The background radiation disturbance is unstable in a dynamic heating environment. So the forenamed discussion about the partition points is the result in the condition of maximal bands number and different partition points. To get the optimal band number, the trade-off between the partition number and the cost function should be considered ${ }^{[14]}$. To this end, the optimal partition points (the optimal band number) is determined by considering the convergence rate of the cost function and the change speed of the convergence rate in the condition of different partition point numbers. The algorithm is shown as follows.

\section{a. Global convergence rate of each partition scheme}

To get a global optimal convergence rates with different partition scheme, the convergence speed is calculated between the $i^{\text {th }}$ cost function and the former $i-1$ cost functions. And the minimal value is defined as the global optimal convergence rate, namely:

$$
g(i)=\min _{0 \leq j \leq i-1}\left\{\frac{I_{N}^{j}(1)-I_{N}^{i}(1)}{i-j}\right\}, \quad i=1,2, \cdots, K
$$

Where $I_{N}^{0}=D_{1, N}$ 。

b. The global optical partition scheme based the change speed between these optimal global convergence rates

The optimal global convergence rates with some specific numbers of partition schemes has the following limit, namely:

$$
\begin{aligned}
& G(i)=g(j), \text { if }\left\{g(j)>\max _{j+1 \leq k \leq K}\{g(k)\}, j \in[1, K-1]\right\}, \\
& X(i)=j, i=1,2, \cdots M
\end{aligned}
$$

With $M$ is the number of specific partition schemes; 
$X$ is used to save the numbers of partition points corresponding the specific partition schemes. In addition to that, the maximal number of partition points is included for a global optimization.

$$
G(M+1)=g(K), \quad X(M+1)=K
$$

The global optimal partition scheme is determined by the change speed of neighboring optimal convergence rates.

$$
i^{*}=\arg \max _{1 \leq i \leq M+1}\{(G(i)-G(i+1))\}
$$

Hence, the optimal number of partition points is $X\left(i^{*}\right)$, the optimal number of partition bands is $\left(X\left(i^{*}\right)+1\right)$, and then the partition points can be located by the $\left(i^{*}\right)^{\text {th }}$ partition case, namely $\left\{M_{i^{*}}(j), j=1,2, \cdots i^{*}\right\}$.

6) the optimal spectral band determination of temperature measurement

The spectral band is chosen to be the optimal spectral one, whose mean value of high frequency ripple within this band is minimal among all of the potential bands when the optimal number of partition points is determined

$$
Q=\left[M_{i^{*}}(l), M_{i^{*}}(l+1)\right]
$$

Where $l=\arg \min _{0 \leq i \leq i^{*}}\left\{\sum_{i=M_{i^{*}}(i)}^{M_{i^{*}}(i+1)}|Y(i)| /\left(M_{i^{*}}(i+1)-M_{i^{*}}(i)\right)\right\}$

\section{Experiment, results and discussion}

\subsection{Experimental Setup}

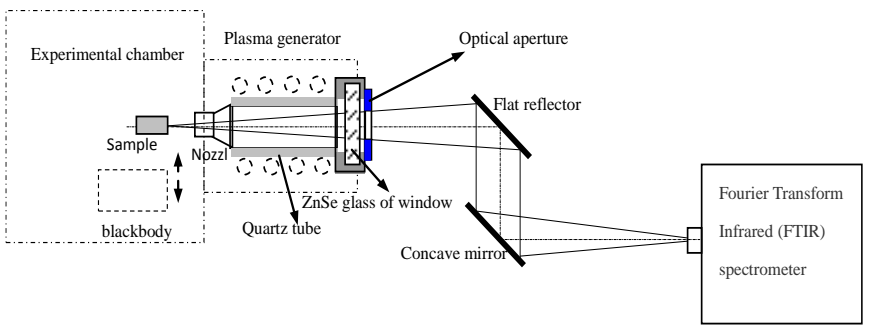

Figure 1 Scheme of thermal radiation measurement system based FTIR spectrometer under plasma wind tunnel In order to validate the above discussed method, the graphite sample, as a reference material, is heated under the dynamical heating condition of plasma wind tunnel. The scheme of thermal radiation measurement system and its experimental setup are given in Fig.. In Fig.1, the plasma is generated through an electric discharging in the gas of quartz tube induced by high frequency alternating current and its corresponding varying electrical field. Then, the plasma is expanded into the experimental chamber and dynamically heat the graphite cylinder sample, whose diameter is $30 \mathrm{~mm}$. The measurement system is consisted of optical aperture, flat reflector, concave mirror and Fourier Transfer Infrared (FTIR) spectrometer. The small and middle-high temperature blackbody is used as the reference by which the measurement system is slightly adjusted to reproduce the original optical route under high temperature blackbody calibration condition. The field diaphragm is used to constraint the measurement spot of the heated sample's front surface. The main test procedure have three steps, i.e. the first step is to measure $S_{b}$, the background radiation when the plasma generator is turn off; then, the second step is to measure $S_{0}$, the background radiation with plasma when the plasma generator is turn on; finally, the sample is moved to the plasma flowfield, and its thermal radiation signal included of above background with plasma, $S$ is measured by FTIR spectrometer. Based these spectral signals from three steps, Eq.1 and

Eq. 2, the proposed adaptive spectral band identification method in noncontact temperature measurement is validated, whose results is given as following.

\subsection{Results of preprocessing the plasma radiation spectrum}

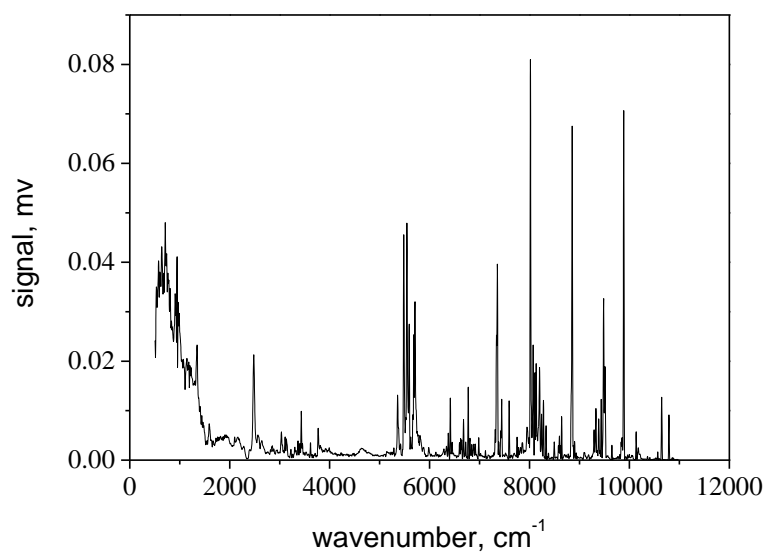

Figure 2 Radiation spectrum of plasma flow field without test model, $\mathrm{S}_{0}$ 


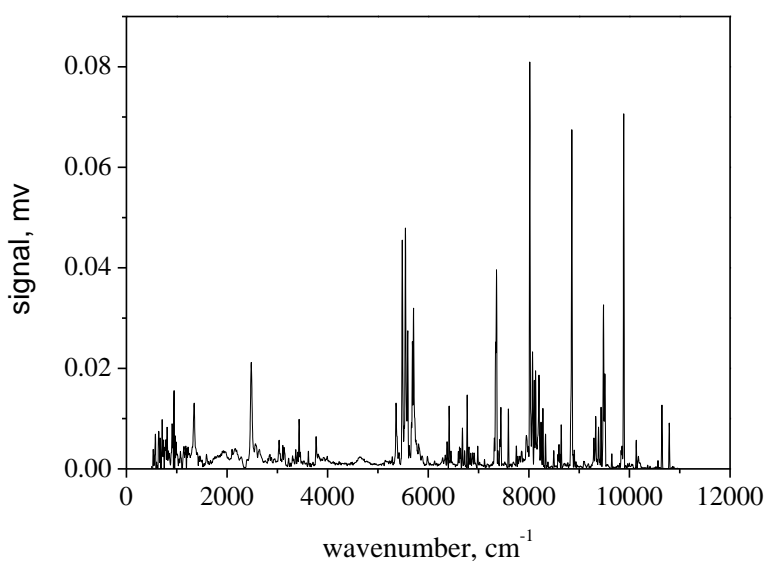

Figure 3 Pure plasma radiation spectrums, $S_{p}$

Fig. 2 shows the radiation spectrum of high-temperature plasma flow, including the ambient background radiation. Fig. 3 shows the radiation spectrum of pure plasma flow. Shown in Fig.2 and 3, the plasma radiation spectrum is discrete and the radiation energy is not uniformity distributed, especially in the near infrared spectrum. Differently, the ambient background radiation spectrum is successive and the energy is intensive in the far infrared spectrum.

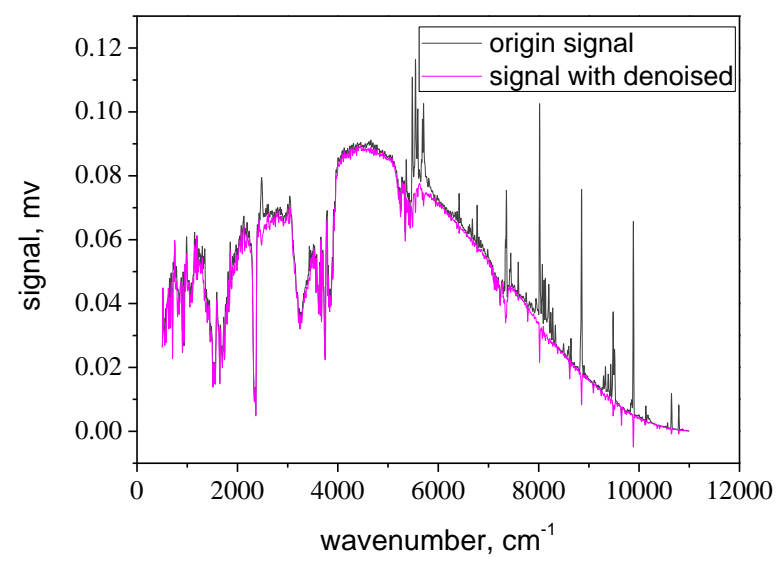

Figure 4 Original spectrum signals with / without plasma

From Fig. 3 and 4, the radiation signal of pure plasma can be effectively separated from the measurement signal of FTIR spectrometer based on Eq. (1) and Eq. (2). And such disturbance can be mostly eliminated from the original radiation spectrum, which means that the noncontact temperature and emissivity are more ef- fectively based on FTIR spectrometer.

\subsection{Adaptive measuring temperature spectral band identification and result analysis}

As shown in Fig. 4, small ripple is still existent in the spectral irradiance though the original spectrum is preprocessed by the above discussed method. So the proposed adaptive spectral band identification method is applied to find the suitable spectral band for calculating the emissivity of the reference material, which is graphite. For a high precision noncontact high temperature measurement, near short wavelength range is desired. Fig. 5 shows the thermal irradiance curve of graphite in the spectral rang of $1.5 \mu \mathrm{m}-3.5 \mu \mathrm{m}$. From Fig. 6, the energy curve has different interferences at different bands, because of plasma disturbances and atmosphere absorption.

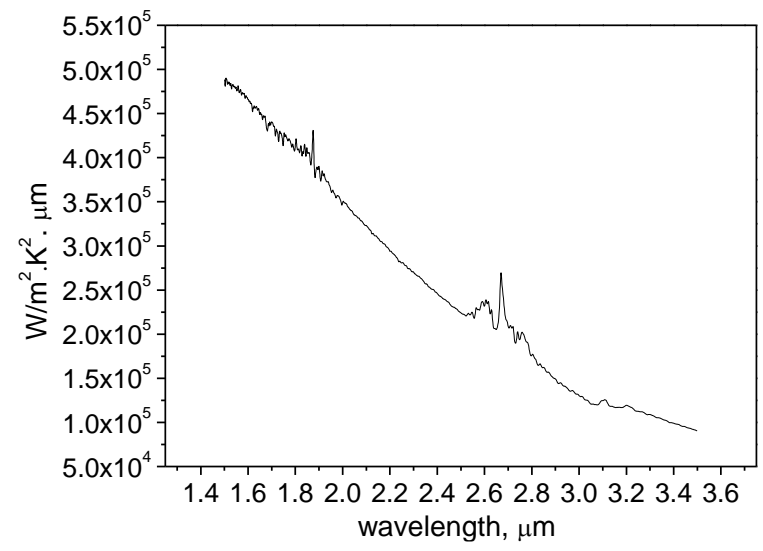

Figure 5 Spectral radiation energy distribution of graphite

Converting the spectrum into a time series, the $4^{\text {th }}$ level high-frequency signal is separated by wavelet analysis. As shown in Fig. 6, the different ripple properties are obvious at different bands. For a higher precision, the band for noncontact temperature measurement should be steady without large ripple as far as possible and the band span should be long enough to avoid the unstable disturbance of plasma. Hence, the multiresolution wavelet analysis is applied to reconstruct high-frequency part of pre-processed signal for identifying the optimal measurement spectral band. 


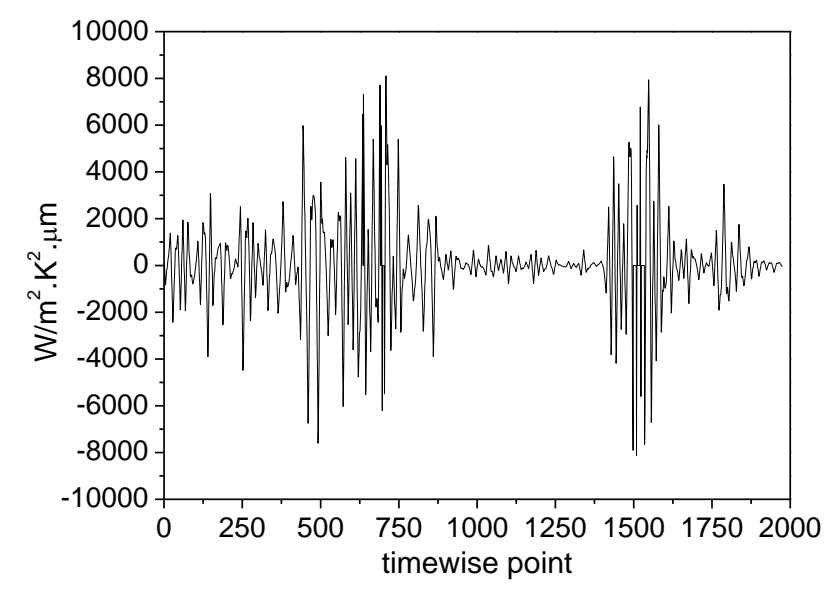

Figure 6 The high-frequency component constructed by the $4^{\text {th }}$ level factors of wavelet

As shown in Fig. 7, the proposed method accurately finds the required band for noncontact temperature measurement and the time series band interval is [804, 1407], which is converted to the corresponding spectral band, $[2.1 \mu \mathrm{m}, 2.5 \mu \mathrm{m}]$.

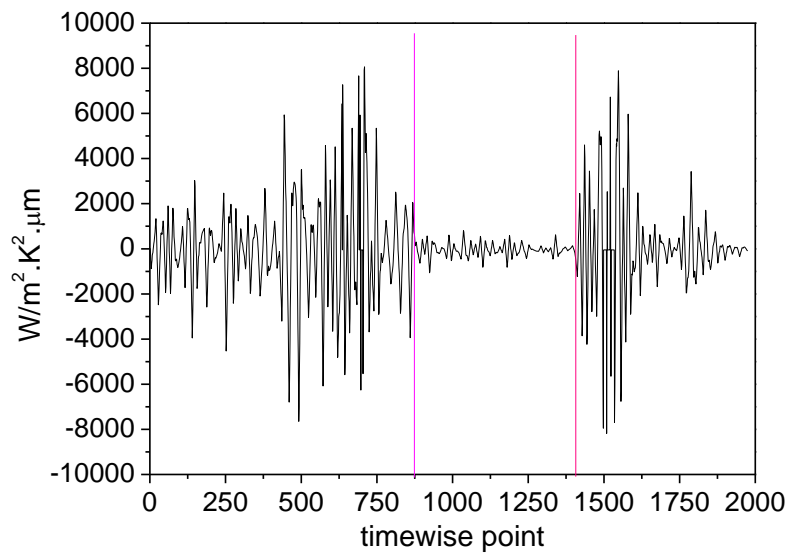

Figure 7 Result of the partition band by the proposed method

The detailed measurement method in Refs.[1], which is involving surface temperature and emissivity of a sample material using the signals collected by FTIR, is applied to calculate thermal radiation parameters of graphite under the dynamic heating environment when the optimal spectral band for noncontact temperature measurement is identified adaptively.

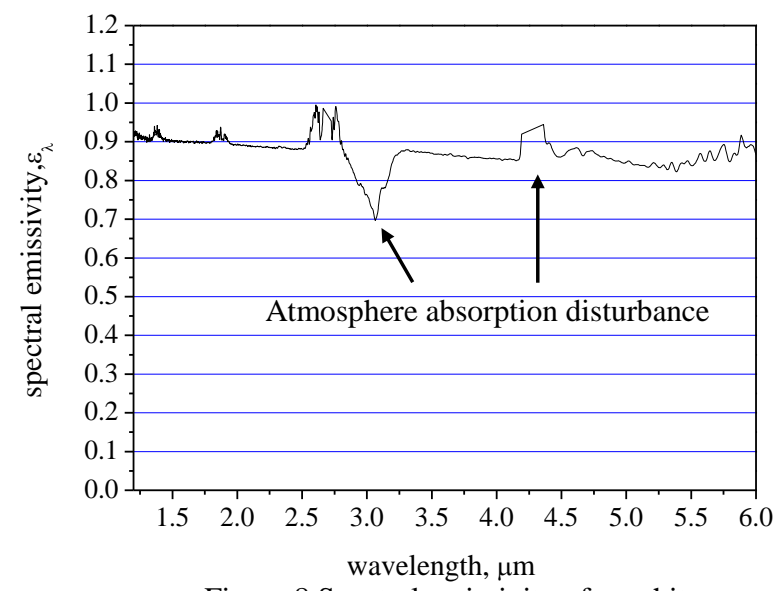

Figure 8 Spectral emissivity of graphite

Fig. 8 shows the measured spectral emissivity of graphite, which coincides with the emissivity distribution of gray-body reference materials, namely the emissivity barely changes with the wavelength. And due to the unstable absorption of atmosphere, some disturbances corresponding to small spectral ranges exist in Fig. 8. But, this disturbances have no obvious influence on the normal total emissivity on wide spectral range, which is dominated by near infrared spectral range when the surface temperature of test model is high enough according to Wien's law.

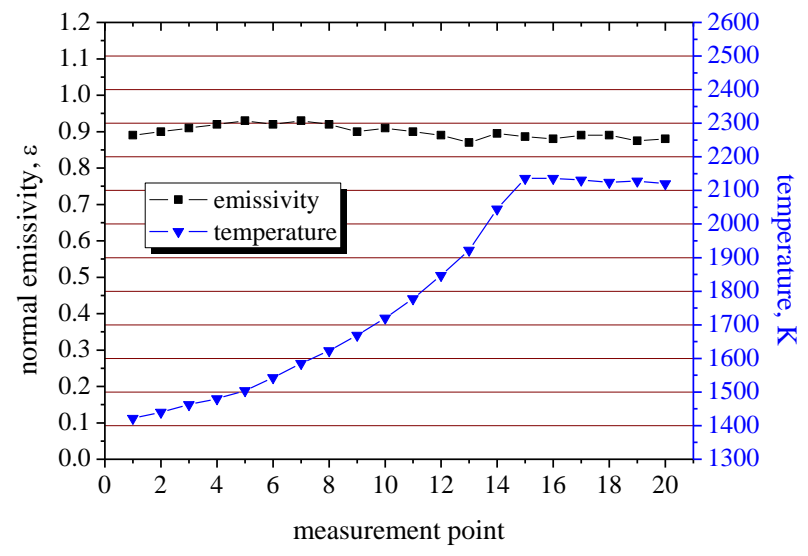

Figure 9 Normal emissivity of graphite under different temperature

Fig. 9 shows the calculated total normal emissivity of graphite. As shown in Fig. 9, the emissivity is about 0.88 . A small descending tendency with rising temperature is observed. Fig. 8 shows that the emissivity slightly declines towards longer wavelengths. The results in this study are consistent with those in Ref. [17]. 
Hence, the adaptive spectral band identification for noncontact temperature measurement based on FTIR spectrometer can be effectively applied to surface temperature and emissivity measurements of the non-ablated heat-proof material under the dynamic heating environment of plasma wind-tunnel.

\section{Conclusion}

In ground testing thermal protection testing, thermal radiation characteristics of non-ablative thermal protection materials are crucial to understand the material behavior. However, the background radiation drastically affects the noncontact temperature measuring method based on FTIR, moreover the emissivity deduced from temperature is also affected. Hence, this study proposes an adaptive spectral band identification method for measuring surface temperature by combining multi-resolution analysis of wavelet with the dynamic programming. The experimental results show that the proposed method can separate the effective spectral band for noncontact temperature measurement with the aid of FTIR. And the results confirm that it is a promising method, which can be applied to obtain surface temperature and emissivity of non-ablating thermal protection materials.

\section{Acknowledgement}

The research is supported by the National Science Foundation of China (NSFC) under grant No. 90716018.

\section{Reference}

[1] Hui Wang, Dejiang Chen, Guolin Wang etc. Measurement technology for material emissivity under high temperature dynamic heating conditions. Measurement Journal, 46(10), (2013) 4023-4031.

[2] B.K. Tsai, R.L. Shemaker, D.P. DeWitt, B.A. Cowans, Z.Dardas, W.N. Delgass, G.J. Dail. Dual-wavelength radiation thermometry: emissivity compensation algorithms [J]. Int. J. Thermophys, 11(1990)
$269-281$.

[3] Yuichiro Tago, Fumie Akimto, Kuniyuki Kitagawa, Norio Arai. Measurement of surface temperature and emissivity by two-dimensional four-color thermometry with narrow bandwidth [J]. Energy, 30 (2-4) (2005) 485-495

[4] J.L. Gardner, T.P. Jones, M.R. Davies. A six-wavelength radiation pyrometer [J]. High Temp. High Press. , 13(1981) 459-466.

[5] B.K. Doloresco. Review of multi-spectral radiation thermometry and development of constrained minimization method [D]. MS thesis, Purdue University, West Lafayette, IN, 1986.

[6] Jingmin Dai, Xinbei Wang, Guibin Yuan. Fourier transform spectrometer for spectral emissivity measurement in the temperature range between 60 and $1500^{\circ} \mathrm{C}$ [J]. Journal of Physics, 13(2005) 63-66.

[7] Claus P. Cagran, Leonard M. Hanssen, Mart Noorma, Alex V. Gura and Sergey N. Mekhontsev. Temperature-resolved infrared spectral emissivity of $\mathrm{SiC}$ and $\mathrm{Pt}-10 \mathrm{Rh}$ for temperatures up to $900^{\circ} \mathrm{C}[\mathrm{J}]$. International Journal of Thermophysics, 28(2) (2007)581-597.

[8] George Teodorescu, Peter D. Jones, Ruel A. Overfelt, Baojian Guo. High temperature, spectral-directional emittance of high purity nickel oxidized in air [J]. J. Mater Sci., 41(2006)7240-7246

[9] I. I. Kovalev, P. G. Muchnik, A. R. Gordon, and V. M. Krylov Application of IKS-14A spectrometer to the measurement of high-temperature emissivity of metals [J]. Measurement Techniques, 13(8) (1970) 1202-1204.

[10] B. Zhang, J. Redgrove, and J. Clark. A transient method for total emissivity determination [J]. International Journal of Thermophysics, $25(2)(2004) 423-438$.

[11] L. Del Campo, R. B. P'erez-S'aez, M. J. Tello, X. Esquisabel, and I. Fern'andez Armco. Iron Normal Spectral Emissivity Measurements [J]. International Journal of Thermophysics, 27(4) (2006)1160-1172.

[12] S. Pidan, M. Auweter-Kurtz, G. Herdrich, and M. Fertig. Recombination Coefficients and Spectral Emissivity of Silicon Carbide-Based Thermal Protection Materials. Journal of Thermophysics and Heat Transfer, 19(4) (2005)566-571.

[13] Bradley, Hax, and Magnanti. Dynamic Programming, chapter 11 in Applied Mathematical Programming. Addison-Wesley,1977. (web.mit.edu/15.053/www/AMP-Chapter-11.pdf)..

[14] Bellman Richard. Dynamic Programming [M]. Dover Publications Inc, 03(2003)1-366. 
[15] .Marc Lavielle. Detection of multiple changes in a sequence of dependent variables [J]. Stochastic Processes and their Applications, 83(1999)79-102.

[16] David F. Walnut . An Introduction to Wavelet Analysis. Birkhäuser, Boston, Jan 27, 2004.

[17] G. Neuer and G. Jaroma-Weiland. Spectral and total emissivity of high-temperature materials [J]. International Journal of' Thermophysic. 19(3) (1998) 917-929. 